\title{
Habituation of the GSR and digital vasomotor components of the orienting reflex as a consequence of task instructions and sex differences
}

\author{
IRVING MALTZMAN, JAY GOULD, OLA J. BARNETT, \\ DAVID C. RASKIN, and CRAIG WOLFF \\ University of California, Los Angeles, Los Angeles, California 90024
}

\begin{abstract}
Two studies are reported in which the effects of task instructions and sex differences on the GSR and digital vasomotor measures of the orienting reflex induced by innocuous words are examined. Instructions to perform an overt response to a tone significantly enhanced the GSR-OR to words preceding the occurrence of the tone, the task signal. No such effect was evident in the digital vasomotor response. A characteristic decline in responsivity to words was evident in the GSR but not in the vasomotor response which was relatively unresponsive. A sex difference, with males manifesting greater GSR responsivity, was found in most phases of the experiments. Less consistent sex differences were observed in the digital vasomotor response.
\end{abstract}

Habituation of the GSR index of the orienting reflex (OR) in normal human adults is complexly determined by prior instructions, predetermining states of the subjects, and individual differences that vary along a variety of dimensions (e.g., Bernstein, Taylor \& Weinstein, 1975; Israel, 1966; Korn \& Moyer, 1968; Maltzman, 1971; Maltzman \& Raskin, 1965; O'Gorman, 1977; Pendery \& Maltzman, 1977; Van Olst, 1971). All of this suggests that, at least at the level of normal adults, habituation of the OR is never a consequence simply of changes in the parameters of the physical stimulus. Habituation is a function of such changes and the state of the participant at the moment where that state is a function of the most complex cortical processes characteristic of human thinking.

The present study examines habituation of the digital vasomotor response (VMR) as well as the GSR index of the $\mathrm{OR}$ as a consequence of task instructions and sex differences. Few studies concerned with the effects of instructions or sex differences have employed more than one measure of the OR.

The experiments reported here were made possible in part by funds provided by the Carnegie Corporation of New York and from Public Health Service Research Grant MH-04684 from the National Institute of Mental Health. For the analyses of the results of the experiments reported here, computing assistance was obtained from the Health Science Facility, UCLA, sponsored by National Institutes of Health Grant FR-3. Jay Gould is now at the University of West Florida. Ola J. Barnett is now at Pep perdine University. David C. Raskin is now at the University of Utah. Requests for reprints should be sent to Irving Maltzman, Department of Psychology, University of California, Los Angeles, California 90024.
Examination of the effects of task variables and sex differences upon two such measures is therefore of some interest.

Habituation phenomena reported here were obtained in the initial phases of each of two experiments where the results of subsequent conditioning have been reported elsewhere (Maltzman, Gould, Barnett, Raskin, \& Wolff, 1977). These experiments investigated differential semantic conditioning of the GSR and digital VMR under five different CS-UCS intervals .5, 5.5, 10.5, 15.5, and $20.5 \mathrm{sec}$. In Experiment 1 , a modified forewarned reaction-time procedure was employed where an innocuous tone/pedalpress complex served as the UCS. Instructions to the students in the experimental groups indicated that they would be required to press a foot pedal in response to a tone. Half the students within each CSUCS interval group were given additional information. They were told that the tone followed a certain word, and they constituted the partially informed (P-I) subgroups as distinguished from the uninformed (U-I) subgroups told only to respond to the tone.

Two control groups were employed in the 10.5sec CS-UCS interval condition in Experiment 1 in addition to the experimental groups. These control groups received the same words and tones as the 10.5-sec experimental groups, but were not instructed to press the pedal to the tone. Group C-I were told that they would hear words and occasional tones. Group C-2 were instructed only that they would hear words; they were given less information about the stimuli to be presented.

The control and experimental groups in the 10.5- 
sec interval provide a partial replication and extension of two of the conditions employed in a previous experiment (Maltzman \& Raskin, 1979). In the latter experiment, a control group was instructed to sit quietly and listen to different words. A pedalpress group were told to press a foot pedal whenever they heard a particular word. Two additional experimental groups were instructed to perfcrm a covert response to a particular word. A habituation session preceded the phase during which the critical word appeared. Task instructions to respond to a particular word produced increased responsivity to all words during habituation, prior to the appearance of the task. Increased GSR-ORs were evoked by all words in the experimental groups as compared to the control group prior to the appearance of the signal for a response. Experiment 1 should enable us to determine whether instructions to respond to a tone would also augment responsivity to words prior to the appearance of the tone signal, or whether increased responsivity was relatively specific to the class of stimuli designated in the task instructions. In the latter case, experimental students would not show enhanced GSRs to words. We could thus determine whether task instructions produce a relatively specific or a nonspecific increase in vigilance or tonic orienting. A nonspecific increase in orienting would be reflected by enhancement in the magnitude of the phasic GSR induced by words when students were instructed to respond to a tone interspersed among different words. Such an effect might come about either because of an overall increase in tonic orienting or because orienting to a tone interspersed among words also requires increased orienting to words as a component of the search for the tone.

Experiment 2 reports the results of a habituation session that preceded a more conventional conditioning experiment where a noxious 110-dB noise served as the UCS (Maltzman et al., 1977). Students in Experiment 2 were told simply to sit quietly and listen to the words and occasional noises. There were no control groups comparable to those employed in Experiment 1. Half the students in the experiment were told that the noise followed a certain word; they constituted the partially-informed (P-I) subgroups, as distinguished from the uninformed (U-I) subgroups told only that there would be words and occasional noises.

Sex differences were examined throughout the course of both experiments, differential conditioning, extinction, and generalization, as well as the initial habituation phase. A variety of different kinds of results have been obtained in studies that have examined sex differences in habituation of the GSR. Most studies have found males to have greater responsivity or resistance to habituation than females
(Kimmel \& Kimmel, 1965; Korn \& Moyer, 1968). In other studies, females have shown greater responsivity, particularly when there was the threat of noxious stimulation (Kopacz \& Smith, 1971; Shmavonian, Miller, \& Cohen, 1968). Still other studies have found that the sex of the subject interacts with the sex of the experimenter (Fisher \& Kotses, 1974). Whether these sex differences are of a general nature, are found in other autonomic measures such as the digital VMR, and are found in situations other than habituation is even less evident than the consistency of the sex differences reported in the studies examining habituation of the GSR.

\section{METHOD}

\section{Subjects}

There were 240 University of California, Los Angeles introductory psychology students who participated in Experiment 1 in partial fulfillment of a course requirement. There were 40 students in each of the five experimental CS-UCS groups and 20 students in each of two control groups. Half the participants in each group were males and half were females. There were 200 students in Experiment 2,20 males and 20 females in each of the five CS-UCS groups.

\section{Procedure}

Details of the procedure have been presented elsewhere (Maltzman et al., 1977). Briefly, words were prerecorded on magnetic tape, played back on a stereophonic tape deck, amplified, and presented via stereophonic headphones.

The GSR was recorded by means of two $20-\mathrm{mm}$ disk electrodes fitted in 5-mm-deep plastic cups which were filled with electrode paste and were attached to the palms of each hand with plastic pressure clips. The VMR transducer was a photoplethysmograph with its photocell face in contact with the skin on the ventral side of the left index finger. A dual-channel Sanborn dc amplifier-recorder was used to record the GSR and VMR.

Habituation consisted of the presentation of 15 common, highfrequency words with an interword interval varying between 24 and $32 \mathrm{sec}$. Conditioning, extinction, and generalization phases followed without interruption. From two to four different neutral control words were interspersed between successive conditioning, extinction, and generalization trials. Conditioning in Experiment 1 consisted of nine presentations of the word "plant", followed by a 70-dB (A) tone to which the students responded with a pedal press. In Experiment 2, a conventional noxious UCS was employed, a .5-sec 110-dB (A) noise. Tests of semantic generalization consisted of a single presentation of the word "stem", embedded among filler words. In Experiment 1, a second test of generalization followed extinction.

The GSR was scored as the largest resistance change occurring within the interval . 5-2.5 sec following each word during habituation, the CS, generalization test word, and neutral control words immediately preceding and following the CS and generalization test words. Digital blood volume changes were recorded in millimeters of pen deflection corrected for recorder sensitivty. The VMR was scored as the largest change in the lowest point of the systolic pulse in the interval .5-2.5 sec and also in the interval 5.0$7.5 \mathrm{sec}$ following a word. Only results from the latter interval will be reported, since the relatively long-latency VMR yielded little data in the first scoring interval. Results reported elsewhere (Maltzman et al., 1977) indicated that the scoring interval employed yielded highly reliable evidence of differential conditioning over a wide range of CS-UCS intervals. 


\section{RESULTS}

Individual differences in the OR were assessed by determining the magnitude of the GSR and the VMR to the presentation of the first word in habituation. The latter was a novel and relatively unexpected stimulus which occurred following a period of silence. Students manifesting a response with a magnitude above the median of the distribution were designated as high orienters, whereas students below the median were designated low orienters. In Experiment 1 , a similar differentiation was made in terms of the distribution of response magnitudes induced by the first tone in conditioning following the habituation series of words. Categorization was made within the smallest cell for each sex within each CS-UCS interval and was determined for the GSR and VMR independently. Thus, the level of OR may serve as a between-subjects factor in the statistical analyses. The .05 level of significance for a two-tailed test was adopted in all statistical tests.

\section{Experiment 1}

Instructions and the GSR. Figure 1 presents the mean magnitude of the GSR during habituation for the two experimental and two control groups receiving the 10.5-sec CS-UCS interval in the subsequent conditioning phase. As previously indicated, Group C-1 were instructed that they were to hear words and tones whereas Group C-2 were told only that they would hear words. Neither group was instructed to respond to the tone. Experimental Group P-I was told that the tone followed a certain word, whereas experimental Group P-U was not. Both experimental groups were instructed to give a pedal response to the tone.

Statistical analyses indicated a significant main ef-

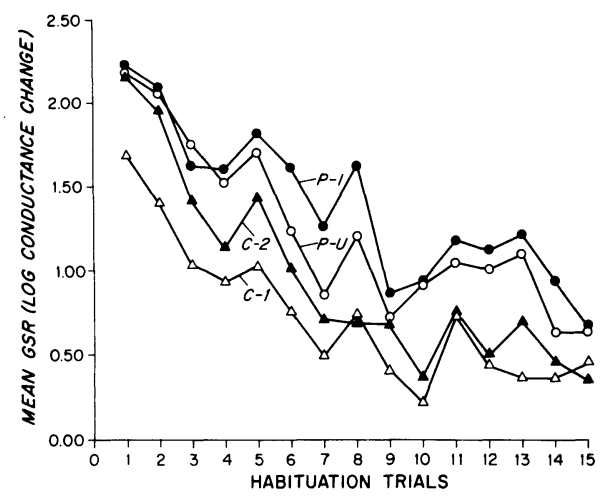

Figure 1. Experiment 1 mean magnitude of the GSR during habituation by groups instructed to perform a pedal-press response and were partially informed, told that the tone followed a certain word, P-I, and uninformed concerning any relationship between the tones and words, P-U. Two control groups did not perform a pedal response, Group $\mathrm{C}-1$ instructed that they would hear tones and words and Group C-2 told only that they would hear words.

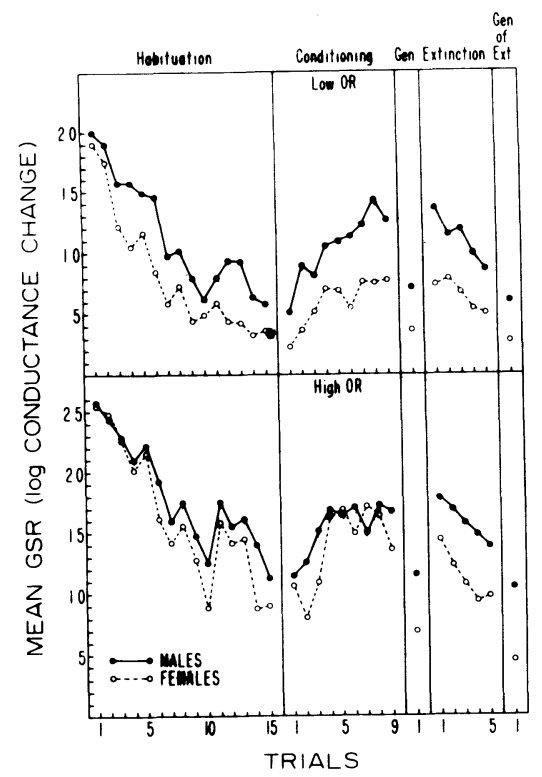

Figure 2. Experiment 1 mean magnitude of the GSR obtained by high- and low-OR males and females during habituation, conditioning, generalization, extinction, and a generalization of extinction test. Results are averaged over CS-UCS intervals, and responses to control words are omitted for the sake of clarity. An innocuous tone-pedal press complex served as the UCS in this modified form of a forewarned reaction time experiment.

fect for OR level and for Trials, and a significant $O R$ by Trials interaction. More important, as suggested by Figure 1, there was a significant main effect due to instructions $\left[\mathrm{F}(1,64)=5.93, \mathrm{MS}_{\mathrm{B}}=4.151\right]$. Individual comparisons indicated that each of the four groups differed significantly from every other. It is important to note, however, that the four groups did not differ significantly in the magnitude of their initial GSR-OR to the first word in habituation.

Analysis of variance and orthogonal polynomials indicated that the basal conductance levels of the four groups did not differ significantly. There were no significant between-group main effects or trends involving the groups. Mean log conductance levels of the experimental groups, P-U and P-I, were 3.11 and 3.11, respectively. Mean log conductance levels for the control groups, C-1 and C-2, were 3.14 and 3.11 , respectively. As in a previous experiment from this laboratory (Maltzman \& Raskin, 1979), effects of task instructions upon GSR responsivity to words prior to the appearance of the task cannot be accounted for by an increase in arousal level. Conductance levels were not increased by the preparation to perform a task required by the experimenter's instructions.

Sex differences in the GSR. Figure 2 depicts the mean magnitude of the GSR for males and for females within high- and low-OR levels during the course of Experiment 1. Responses to control words preceding and following the CS and generalization 
test words have been omitted from the figure for the sake of clarity.

Again, it must be noted that males and females did not differ in their initial GSR-ORs. They did differ significantly in overall rsponsivity during habituation $[\mathrm{F}(1,160)=11.08]$. Also significant was OR level $\left[\mathrm{F}(1,160)=102.89, \mathrm{MS}_{\mathrm{B}}=3.962\right]$. Males and females were significantly different during conditioning $[\mathrm{F}(1,128)=9.84]$, as was OR level $[\mathrm{F}(1,128)=35.65$, $\left.\mathrm{MS}_{\mathrm{B}}=7.991\right]$. The sex difference was also significant on the generalization test trial $[\mathrm{F}(1,160)=5.26]$, as was OR level $\left[\mathrm{F}(1,160)=18.45, \mathrm{MS}_{\mathrm{B}}=1.326\right]$. The same differences were reliably obtained in extinction [sex, $\mathrm{F}(1,160)=18.37$, and OR level, $\mathrm{F}(1,160)=$ 22.14, $\left.\mathrm{MS}_{\mathrm{B}}=5.431\right]$. Comparable results were obtained in the generalization of extinction test [sex, $F(1,160)=19.87$, and OR level, $F(1,160)$ $\left.=19.34, \mathrm{MS}_{\mathrm{B}}=1.390\right]$. The degrees of freedom differ for the statistical tests conducted during conditioning and the other phases because the .5 -sec interval group was omitted in the former phase. It is important also to note that the obtained sex differences were all between-subject differences. A sex difference was present over all words. There were no significant Sex by Words interactions in any of the experimental phases following habituation where such an interaction might occur. Absence of the latter interaction indicates that males and females did not differ in differential semantic conditioning, generalization, or extinction. They differed consistently in overall responsivity, with males showing greater responsivity than females following their initial GSR-OR to the first word in habituation, which did not manifest a sex difference.

Task instructions, habituation, and sex differences in the VMR. Results obtained during the habituation phase of Experiment 1 indicated that habituation of the digital VMR was quite different in nature from habituation of the GSR. Control and experimental groups in the 10.5-sec CS-UCS interval, those instructed to respond to the tone and those told only to listen, did not differ in the magnitude of their VMRs as they did in the case of the GSR.

In order to examine changes in the VMR in greater detail, the students were categorized as high- and loworienters in terms of the magnitude of the VMR to the first tone as well as the first word. Use of the response to the first tone permits a measure of the OR to a novel stimulus idependent of the response evoked during the course of habituation. Figure 3 depicts the course of habituation of the VMR to words where high- and low-OR students were categorized on the basis of the magnitude of their VMR to the first tone presented during the subsequent conditioning phase.

Results of the statistical analyses revealed a significant between-subjects effect for OR level $[F(1,160)$

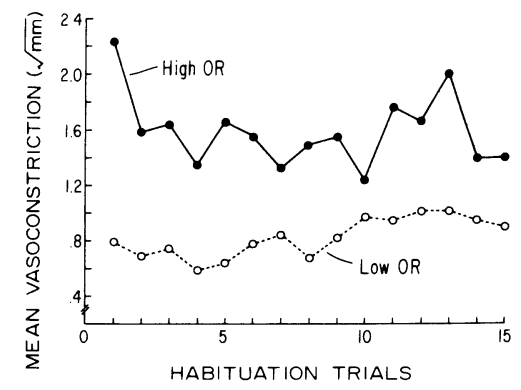

Figure 3. Experiment 1 mean digital vasoconstriction obtained by high- and low-OR students during habituation. Levels of $O R$ were established on the basis of the response magnitude to the first tone in the conditioning phase that followed habituation.

$\left.=57.68 \mathrm{MS}_{\mathrm{B}}=7.544\right]$. Significant within-subject effects were obtained for Trials and the OR by Trials interaction $[\mathrm{F}(14,2240)=3.85$, and $\mathrm{F}(14,2240)$ $=2.88$, respectively; $\mathrm{MS}_{\mathrm{W}}=1.444$ ]. A more revealing assessment of the response trends stems from an analysis of orthogonal polynomials.

The latter analysis indicatd that the trends for high- and low-OR students differed significantly $\left[\mathrm{F}(1,160)=7.48, \mathrm{MS}_{\mathrm{B}}=2.564\right]$. Mean $\mathrm{L}$ scores for high- and low-OR students were -.20 and 42 , respectively, indicating that there was a decline in the response trend for the high-OR students and an increase in the response trend for the low-OR students. There appeared to be a regression towards the mean. Comparable results were obtained when OR levels were established by categorizing the students in terms of the magnitude of their initial VMR to the first word in habituation.

A significant sex difference was obtained during habituation $\left[\mathrm{F}(1,160)=12.69, \mathrm{MS}_{\mathrm{B}}\right.$ 7.544]. Males showed greater responsivity than females, with no difference in the magnitude of the initial VMR-OR induced by the first word in the habituation series.

During conditioning, a significant Sex by Words interaction occurred $\left[\mathrm{F}(2,256)=10.53, \mathrm{MS}_{\mathrm{W}}=\right.$ 1.964]. Mean magnitude of the VMRs obtained by males for $C_{1}, C S$ and $C_{2}$ words were $1.55,2.17$, and 1.22, respectively. Mean magnitude of the VMRs obtained by females for $\mathrm{C}_{1}, \mathrm{CS}$, and $\mathrm{C}_{2}$ were 1.11 , 1.41 , and .99 , respectively. Thus, a discrepancy in the results obtained by the GSR and VMR measures of the OR is apparent. Both measures showed overall larger responses to all words on the part of males as compared to females. But the VMR showed superior differential semantic conditioning on the part of males as well. It is obviously important to have within-subject control stimuli such as provided by differential conditioning in order to adequately interpret a sex difference apparent in a given response measure. In the absence of differential conditioning, control words as well as a CS word, the betweensubjects difference in the GSR could be interpreted 
as a superiority in conditioning, whereas it indicated only greater responsivity in the GSR mode.

Only the main effect for sex was significant during extinction $\left[\mathrm{F}(1,160)=23.66, \mathrm{MS}_{\mathrm{B}}=7.561\right]$. There was no further evidence of differential resistance to extinction or generalization as a function of sex differences.

\section{Experiment 2}

The GSR. It may be recalled that, in contrast to Experiment 1, the present experiment employed a conventional, noxious UCS. Variations in instructions provided experimental and control groups are absent in the present experiment. There was, however, a slight variation in the amount of information given to the students. Half the students were told that the noise followed a certain word, and half were not. This variation in instructions did not produce a difference during habituation or the subsequent conditioning, generalization, or extinction phases of the experiment (Maltzman et al., 1977).

Figure 4 shows the mean magnitude of the GSRs in Experiment 2 produced by males and females within the high- and low-OR levels during habituation, conditioning, generalization, and extinction. A significant sex difference was not present during habituation, but was in each of the remaining phases of the experiment. During conditioning, the sex difference yielded $\mathrm{F}(1,128)=12.50, \mathrm{MS}_{\mathrm{B}}=4.955$; during generalization, $\mathrm{F}(1,160)=8.73, \mathrm{MS}_{\mathrm{B}}=.305$ and

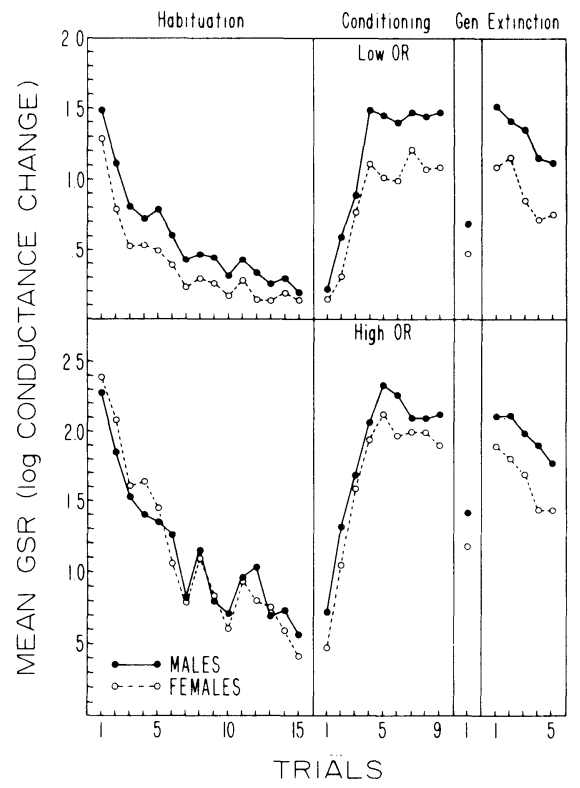

Figure 4. Experiment 2 mean magnitude of the GSR obtained by high- and low-OR males and females during habituation, conditioning, generalization, and extinction. Results are averaged over CS-UCS intervals and responses to control words are omitted for the sake of clarity. A noxious noise served as the UCS in this experiment.

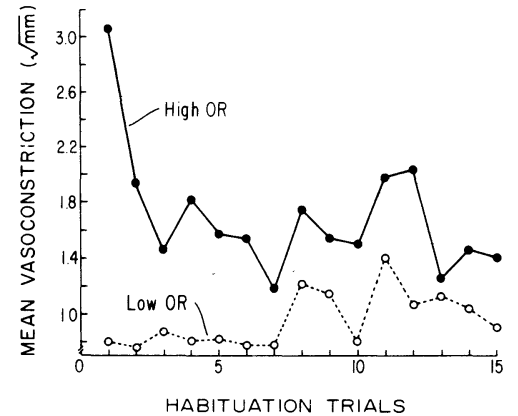

Figure 5. Experiment 2 mean digital vasoconstriction obtained by high- and low-OR students during habituation. Levels of $O R$ were established on the basis of the response magnitude to the first word in habituation.

during extinction, $\mathrm{F}(1,160)=14.55, \mathrm{MS}_{\mathrm{B}}=3.152$. Level of OR was significant in all phases of the experiment. Again, a sex difference was not present in the magnitude of the GSR to the first word in habituation and was not significant during the habituation series as a whole. Significantly greater responsivity on the part of males, as compared to females, emerged as the experiment progressed. Figure 4 indicates that despite the presence of a highly noxious UCS the maximum magnitude of the GSR-CR did not exceed the magnitude of the GSR evoked at the outset of the experiment.

It appears that the students in Experiment 1 were more responsive than those in Experiment 2, as suggested by the greater magnitude of their initial GSROR. High-OR students conditioned with a noxious UCS in Experiment 2 exceeded the magnitude of the GSR-CR attained by the more responsive high-OR students conditioned with the innocuous UCS in Experiment 1. However, the magnitude of the GSRCRs of the students in Experiment 2 did not exceed the magnitude of the GSR-OR evoked in Experiment 1 or their own initial GSR-OR.

The VMR. Figure 5 shows the mean magnitude of the VMR evoked by the different words in the habituation phase with students categorized as highor low-OR participants on the basis of the magnitude of their VMR to the first word in habituation. Significant effects were obtained for OR level $[\mathrm{F}(1,160)$ $\left.=49.15, \mathrm{MS}_{\mathrm{B}}=8.517\right]$, Trials $\mathrm{F}(14,2240)=$ 6.03], and the $O R$ by Trials interaction $[F(14,2240)$ $\left.=6.26, \mathrm{MS}_{\mathrm{W}}=1.985\right]$. Orthogonal polynomial analysis indicated that the linear components of the OR-level response trends were also significant $\left[\mathrm{F}(1,160)=25.98, \mathrm{MS}_{\mathrm{B}}=.204\right]$. Mean L scores for high- and low-OR students were - .21 and .12, respectively. The latter scores reflect the initial decline in the VMR for high-OR students and the subsequent increase in trend for low-OR students. Quadratic components of the OR trends were also significant, indicating a significant difference in curvature in the two 
trends $\left[\mathrm{F}(1,160)=2.71, \mathrm{MS}_{\mathrm{B}}=.144\right]$. Finally, there was a significant difference in the cubic components of the trends for the two groups $[\mathrm{F}(1,160)=12.32$, $\left.\mathrm{MS}_{\mathrm{B}}=.135\right]$, probably produced by the cyclic decline and rise in the trend for the high-OR students. Again, as in Experiment 1, there is relatively little habituation evident, particularly if high- and lowOR subgroups are collapsed. There is little evidence of habituation, a monotonic decline in responsivity, because there was little that could habituate to begin with.

No significant between- or within-subject VMR sex differences were obtained in habituation, conditioning, or generalization in the present experiment. In contrast, such differences were obtained in Experiment 1. There was, however, a significant between-subjects effect for sex during extinction $[F(1,160)=5.22$, $\left.\mathrm{MS}_{\mathrm{B}}=11.246\right]$. Males displayed overall greater VMR responsivity than females. Mean magnitude of the VMR for males and females were 1.65 and 1.37, respectively.

\section{DISCUSSION}

Results from Experiment 1 showing the effects of task instructions on the GSR during habituation prior to the occurrence of the task-relevant stimulus corroborate and extend previous findings (Maltzman \& Raskin, 1979). Experimental groups instructed to perform an overt response to a tone showed significantly larger GSR magnitudes to innocuous words prior to the first appearance of the tone than did control students not given the reaction time paradigm instructions. Heightened GSR reactivity, presumably reflecting orienting activity, occurred to words even though the instructions were to respond to a tone. The latter, of course, did not occur until after the habituation phase examined here was completed. In the present experiment, a tone was imparted with significance as the signal for the reaction time task by instructions at the start of the experiment. In a previous study (Maltzman \& Raskin, 1979), a particular word was made the signal for the reaction time task, and this resulted in heightened responsivity to all words prior to the appearance of the significant word. The present result indicates that a relatively nonspecific effect was obtained. However, basal conductance level, a commonly employed index of arousal, did not differ significantly among the various groups. Thus, O'Gorman's (in press) attempt to account for increases in orienting in the absence of stimulus change in terms of available concepts such as arousal level is contradicted by the present results.

Results of the kind obtained in the present experiment formed the bases, in part, for the hypothesis that the OR is not only a function of neuronal models, stimulus change, and traditional concepts such as arousal. The dominant focus is among the most important additional conceptions necessary to account for the variety of experimental effects upon expressions of ORs that may be seen when task instructions are varied (Maltzman, in press). An adequate account of results such as those obtained in the present study, as well as others (Maltzman \& Raskin, 1979), requires a conception of a dominant focus or cortical set, a heightened selective influence upon the expression of ORs. Degree of specificity, the extent of the set effect, of task instructions upon the GSR expression of the OR, is a problem that must be explored by further research.

An important theoretical implication is suggested by the finding that the difference in magnitudes of the GSR induced as a function of differential task instructions developed as the habituation series of words progressed. A significant difference among the subgroups did not occur in the responses to the first word. Absence of a difference in responses to the first stimulus, with its subsequent development as habituation progressed, suggests that the initial GSR-OR to an unpredictable, surprising stimulus induces a relatively involuntary OR. Repeated presentations of a given stimulus or class of stimuli where the class is predictable provides the opportunity for differential higher cortical activity that may lead to differential amounts and rates of habituation. This suggests that, as habituation progresses, a somewhat different kind of OR is reflected by the GSR, one that may be called a voluntary, as distinguished from the initial involuntary, OR (Maltzman, 1971; 1977). Results produced by the different kinds of task instructions during habituation in Experiment 1 indicate that different kinds of instructions, experimenter-induced and presumably also self-induced, predetermine, to a great extent, the kind and amount of OR activity manifested by the human observer. Habituation of the GSR-OR is not only a function of parameters of physical stimuli and their temporal relations, but is fundamentally a consequence of prior established cortical sets or predetermining instructions, either experimenter- or self-induced. Different kinds of instructions from those employed in the present experiments, instructions establishing a different kind of cortical set, can radically modify the initial GSR-OR to a novel stimulus as well as subsequent stimulus presentations (Korn \& Moyer, 1968; Pendery \& Maltzman, 1977).

Just as the effects of the task instructions administered to the students do not manifest themselves in the initial GSR-OR, so too the sex difference in the GSR in Experiment 1 and 2 was not present in the initial GSR-OR to the first word in the habituation series. Absence of a sex difference in the initial GSR- 
OR and its subsequent development suggests that the sex difference, when it appears, is a function at least in part, of different kinds of cognitive styles or cortical sets displayed by males and females. Sex differences found in the present experiments appear in the voluntary rather than the involuntary GSR-OR. Presumably, the voluntary OR is to a greater extent under verbal regulation mediated by the frontal cortex than the involuntary $O R$ induced primarily by novel and nonsignificant stimuli (Luria, 1973).

Since the voluntary GSR-OR shows a sex difference and not the involuntary GSR-OR, the various interactions obtained between sex and task in the present experiments as well as in other studies (e.g., Fisher \& Kotses, 1974) are not completely puzzling. These interactions are largely a consequence of sex differences in cognitive styles or patterns of thinking about the different situations that are a consequence of the different kinds of past experiences acquired by males and females. A basic, unsolved, problem is the nature of these different styles and kinds of thinking displayed by males and females that produce differences in the voluntary OR reflected as different kinds of GSR activity.

An alternative interpretation may be possible in terms of the sex differences in adrenergic-cholinergic balance that manifest themselves in an interaction with different types of cognitive tasks (Broverman, Klaiber, Kobayashi, \& Vogel, 1968).

Differences between the digital VMR and the GSR occurred in both experiments in terms of task instructions and sex differences, and clearly were not predictable. It appears that the VMR is a relatively insensitive measure of the OR to innocuous words, and apparently to nonsignificant and innocuous stimuli generally (e.g., Ginsberg \& Furedy, 1974; Ray, Piroch, \& Kimmel, 1977). It did, however, manifest differential semantic conditioning (Maltzman et al., 1977) in both the forewarned reaction time paradigm and with a conventional noxious UCS. It also reflected a significant sex difference in differential conditioning in Experiment 1, the forewarned reaction time paradigm, whereas the GSR did not. But the VMR did not reflect the effects of task instructions as did the GSR in Experiment 1. There is a relative absence of responsivity of the VMR to innocuous stimuli and therefore an absence of an habituation effect.

Analyses of levels of VMR-OR both in terms of the initial response to the tone in Experiment 1 and the initial response to the first word in habituation in Experiment 2 cast some light on the problem of lack of habituation to innocuous words found in the present experiments. Students below the median in the magnitude of their VMR-OR, whether it was to the first word or the first tone, showed very little initial responsivity. They showed a slight increase on subsequent trials. High-VMR-OR students did show response decrements. Averaging the two groups, the usual procedure, gives the appearance of little, if any, habituation for the group as a whole. Habituation of the GSR as a function of level of the GSR-OR, in contrast, does not display a regression towards the mean of the group. Students above and below the median GSR-OR tend to show parallel response trends of a decreasing nature so that the average habituation curve reflects the decreasing trends for high and for low responders.

Such differences between two measures presumed to be indices of the OR emphasize the complexity of the theoretical problem involved in attempts to explain the nature of orienting activity (Furedy, 1968; Ginsberg \& Furedy, 1974).

\section{REFERENCES}

Bernstein, A. S., Taylor, K. W., \& Weinstein, E. The phasic electrodermal response as a differentiated complex reflecting stimulus significance. Psychophysiology, 1975, 12, 148-169.

Broverman, D. M., Klaiber, E. L., Kobayashi, Y., \& VOGEL, W. Roles of activation and inhibition in sex differences in cognitive abilities. Psychological Review, 1968, 75, 23-50.

Fisher, L. E., \& Kotses, H. Experimenter and subject sex effects in the skin conductance response. Psychophysiology, 1974, 11, 191-196.

FUREDY, J. J. Human orienting reaction as a function of electrodermal versus plethysmographic response modes and single versus alternating stimulus series. Journal of Experimental Psychology, 1968, 77, 70-78.

Ginsberg, S., \& Furedy, J. J. Stimulus repetition, change, and assessments of sensitivites of and relationships among an electrodermal and two plethysmographic components of the orienting reaction. Psychophysiology, 1974, 11, 35-43.

ISRAEL, N. R. Individual differences in GSR orienting response and cognitive control. Journal of Experimental Research in Personality, 1966, 1, 244-248.

Kimmel, H. D., \& Kimmel, E. Sex differences in adaptation of the GSR under repeated applications of a visual stimulus. Journal of Experimental Psychology, 1965, 70, 536-537.

Kopacz, F. M., \& Smith, B. D. Sex differences in skin conductance measures as a function of shock threat. Psychophysiology, 1971, 8, 293-303.

KorN, J. H., \& MoYer, K. E. Effects of set and sex on the electrodermal orienting response. Psychophysiology, 1968, 4, 453-459.

Luria, A. R. The working brain. New York: Basic Books, 1973.

Maltzman, I. Orienting reflexes and significance: A reply to O'Gorman. Psychophysiology, in press.

Maltzman, I. The orienting reflex and thinking as determiners of conditioning and generalization to words. In H. H. Kendler \& J. T. Spence (Eds.), Essays in neo-behaviorism: A memorial volume to Kenneth $W$. Spence. New York: Appleton-CenturyCrofts, 1971.

Maltzman, I., Gould, J., Barnett, O. J., Raskin, D. C., \& Wolff, C. Classical conditioning components of the orienting reflex to words using innocuous and noxious unconditioned stimuli under different conditioned stimulusunconditioned stimulus intervals. Journal of Experimental Psychology: General, 1977, 106, 185-212.

Maltzman, I., \& Raskin, D. C. Effects of individual differences in the orienting reflex on conditioning and complex processes. Journal of Experimental Research in Personality, 1965, 1, 1-16. 
Maltzman, I., \& Raskin, D. C. Selective orienting and habituation of the GSR as a consequence of overt and covert activity. Physiological Psychology, 1979, 7, 204-208.

O'Gorman, J. G. The orienting reflex: Novelty or significance detector? Psychophysiology, 1977, in press.

Pendery, M., \& Maltzman, I. Instructions and the orienting reflex in "semantic conditioning" of the galvanic skin response in an innocuous situation. Journal of Experimental Psychology: General, 1977, 106, 141-171.

Ray, R. L., Piroch, J. F., \& Kimmel, H. D. The effect of task and stimulus variability on habituation of electrodermal and vasomotor reaction. Physiological Psychology, 1977, 5, 189-196.

Shmavonian, B. M., Miller, L. H., \& Cohen, S. I. Differences among age and sex groups in electrodermal conditioning. Psychophysiology, 1968, 5, 119-131.

VAN Olst, E. H. The orienting reflex. The Hague: Mouton, 1971.

(Received for publication February 1, 1978; revision accepted January $9,1979$. 